\title{
Relationship between oxidative stress and haematological indices in patients with diabetes in the Ghanaian population
}

RH Asmah ${ }^{1 *}$, G Yeboah ${ }^{1}$, H Asare-Anane ${ }^{2}$, S Antwi-Baffour ${ }^{1}$, TN Archampong ${ }^{3}$, CA Brown ${ }^{1}$, G Amegatcher ${ }^{1}$, DN Adjei ${ }^{1}$, B Dzudzor ${ }^{4}$, J Akpalu ${ }^{3}$ and PF Ayeh-Kumi ${ }^{1}$

\begin{abstract}
Background: Persistent hyperglycaemia is a hallmark of Diabetes Mellitus (DM). It causes increased production of free radicals, especially reactive oxygen species (ROS), - resulting in oxidative stress. Reactive Oxygen Species have been implicated in the development of haematological complications in patients with diabetes. Superoxide Dismutase (SOD) is one of the most effective antioxidant enzyme defense systems against free radicals.

Methods: From February through May 2014, we assessed the relationship between oxidative stress and haematological profiles among individuals with and without diabetes. A cross sectional study of 66 case patients and 44 age-matched controls were recruited from the National Diabetes Management and Research Centre (NDMRC), Korle-Bu Teaching Hospital, Accra, Ghana. Blood samples were obtained from study participants with consent. We determined the haematological profiles of study participants and measured their oxidative stress levels using a standardized kit for SOD activity.

Results: Higher white blood cell (WBC) counts were seen in the diabetes cohort ( $p$-value $=0.023$ ). The SOD activity tended to be lower in diabetes patients ( $p$-value $=0.144$ however) while higher neutrophil levels seemed to correlate with SOD activity $\left(R=0.249 ; R^{2}=6.2 \% ; p\right.$-value $\left.=0.049\right)$. There did not appear to be a correlation between fasting blood glucose (FBG) and SOD activity $(R=-0.044$; $p$-value $=0.727)$.

Conclusion: The study reports similar oxidative stress levels, as measured by SOD activity, in diabetic and non-diabetic adults. The SOD activity did not appear to correlate with FBG and several other haematological parameters. Further study would be required to investigate the relationship between these haematological indices and diabetic micro- and macro-vascular complications in our population.
\end{abstract}

Keywords: Reactive oxygen species, Superoxide dismutase, Diabetes Mellitus, Haematological parameters

\section{Background}

Diabetes mellitus (DM) is major cause of adult morbidity and death in Ghana - with an estimated prevalence of $3.3 \%$ that represents a diabetic population of 354,020 [1]. The disease is characterized by a deficiency in insulin resulting in disturbances in carbohydrate, lipid and protein metabolism, which manifest as chronic hyperglycaemia. Persistent hyperglycaemia in DM causes an increased production of free radicals especially Reactive Oxygen Species (ROS). Destruction mediated by such

\footnotetext{
* Correspondence: rhasmah@chs.edu.gh

'Department of Medical Laboratory Sciences, School of Biomedical and

Allied Health Sciences, University of Ghana, Korle-bu, Accra, Ghana

Full list of author information is available at the end of the article
}

free radicals results in protein denaturation, DNA strand breaks and alterations of cell membrane fluidity. Antioxidant enzyme defense systems against free radicals include superoxide dismutase (SOD) and catalase $[2,3]$. Superoxide Dismutase is one of the most effective intracellular enzymatic antioxidants that catalyzes the conversion of superoxide $\left(\mathrm{O}_{2}^{-}\right)$to oxygen $\left(\mathrm{O}_{2}\right)$ and to the less reactive species, hydrogen peroxide $\left(\mathrm{H}_{2} \mathrm{O}_{2}\right)[4,5]$. Reports indicate that antioxidants are either reduced or not sufficient to eliminate ROS in diabetic patients leading to oxidative stress [6]. Oxidative stress results from an imbalance between free radicals and the body's antioxidant defense systems; resulting in red blood cell dysfunction, platelet destruction and tissue injury. In patients 
with diabetes, such abnormalities may affect the functions of blood cells as well as the coagulation system leading to complications such as anaemia, infection, and hypercoagulability [7]. Yet, these haematological changes also opens up the possibility that circulating blood cells, particularly leukocytes including lymphocytes and neutrophils could serve as biomarkers for oxidative stress, and as an index of diagnostic significance in routine clinical evaluation of the state of diabetes [8]. We conducted this study with a two-fold primary objective. First, to determine whether patients with diabetes have higher oxidative stress, and second to evaluate the association between oxidative stress and certain changes in hematological indices.

\section{Methods}

\section{Study site}

This study was carried out at the National Diabetes Management and Research Centre (NDMRC), Accra, Ghana. It serves as the Diabetes Clinic of the Korle-Bu Teaching Hospital (KBTH). The KBTH is a 2000-bed tertiary teaching hospital with about 200 admissions per day. The hospital covers all medical specialties and provides referral healthcare services to an estimated population of 24 million Ghanaians. The NDMRC is designed to manage diabetic conditions of patients in Ghana. The Centre also supports and enhances the national effort in diabetes, its complications, and related endocrine and metabolic diseases through interdisciplinary research.

\section{Study population}

The study population comprised Diabetes Mellitus patients aged 30 to 80 years who attended the NDMRC and consented to participate in the study. A volunteer group without Diabetes Mellitus and of the same agebrackets were enrolled as controls.

\section{Ethical approval}

Ethical approval was obtained from the Ethics and Protocol Review Committee of the School of Allied Health Sciences, University of Ghana prior to the enrolment of participants.

\section{Study design}

This was an analytical cross-sectional study with agematched comparison controls. The study was conducted from February through May 2014. Two sampling approaches were used. First, an active case-finding network was organized with visits to the NDMRC at Korle-Bu. Physician-identified patients with known type-1 or type2 diabetes attending the center for review and management of various diabetic conditions were referred to the research team for screening and inclusion in the study after informed consent was obtained. Diabetic patients enrolled into the study were categorized as cases. Prior to enrollment, the nature, purpose and potential risks of the study were explained to all subjects. Patients' case history was ascertained through physician-assisted review of medical folders. Cases were recruited based on the following screening criteria: $30-80$ years of age, preprandial capillary plasma glucose levels of $3.9-7.2 \mathrm{mmol} /$ 1 and mean plasma glucose of $8.6 \mathrm{mmol} / \mathrm{l}$ correlating with achievement of target glycated haemoglobin (HbA1c) of $<7 \%$ as per the [9] criteria. Overall 66 patients were enrolled to participate in the study. Forty-four other subjects without diabetes were prospectively recruited as controls within the same study period. The control group were aged-matched volunteers accompanying cases to the diabetic center. The same biochemical criteria were examined in the controls subjects. Controls had fasting plasma glucose $<5.6 \mathrm{mmol} / \mathrm{L}$ or two-hour glucose during OGTT $<7.8 \mathrm{mmol} / \mathrm{L}$. Second, venous blood samples were collected from diabetics and nondiabetic controls and analyzed for various haematological changes.

\section{Exclusion criteria}

Pregnant and lactating women were excluded from the study. Patients with medical conditions such as infections, cerebrovascular diseases, ischaemic heart disease and malignancies excluded from the study. Other exclusion criteria were smoking and alcoholism.

\section{Study conduct}

Haematological indices and fasting glucose analysis

After an overnight fast, $5 \mathrm{ml}$ of venous blood was collected from each participant. Three milliliters of which was put into an EDTA tube for haematological indices and SOD activity analysis and $2 \mathrm{ml}$ was put into a fluoride tube for fasting plasma glucose (FPG) analysis using an automated analyzer (A25 Biosytems, Canada). The haematological indices were determined by performing a full blood count (FBC) using an automated haematology analyzer (Sysmex KX-21 N, Germany). The following haematological indices were assessed: Haemoglobin, Haematocrit, Mean cell volume, white cell count (including lymphocyte and neutrophil count); platelet count. The rest of the blood sample was then centrifuged at $4000 \mathrm{rpm}$ for $10 \mathrm{~min}$ to separate plasma from the red blood cells. The stored red blood cells were used for further work involving SOD activity determination.

\section{Measurement of sod activity as an index of oxidative stress} status

Briefly, the SOD activity levels were determined by using SOD Assay kit (Cayman Chemicals, Michigan, USA). Whole blood obtained by venipuncture from the participants was centrifuged at $4000 \mathrm{rpm}$ for $10 \mathrm{mins}$, and plasma carefully separated. Two hundred and fifty (250) 
microliters of erythrocytes obtained from the packed cells was lysed with thousand $(1,000)$ microliters of icecold deionized distilled water and centrifuged at $6,000 \mathrm{rpm}$ for $20 \mathrm{~min}$. Thousand microliters $(1000 \mu \mathrm{l})$ of the supernatant (erythrocyte lysate) was collected for assay and stored on ice. The supernatant fluid was then diluted by a factor of 100 with sample buffer, and $10 \mu \mathrm{l}$ of the diluted solution used to assay $\mathrm{Cu} / \mathrm{ZnSOD}$ (Copper/Zinc Superoxide dismutase) activity as described by the kit manufacturer.

\section{Data analysis}

Data obtained was put into a database and analyzed statistically using Statistical Package for Social Sciences (SPSS) 20.0 software for windows. Summary statistics was presented using the descriptive statistics of mean and standard deviation for numerical variables and proportions for categorical variables. Independent t-test or analysis of variance (ANOVA) and Pearson's correlation analysis were used to determine the mean differences and the correlation between oxidative stress and haematological parameters respectively. A $p$-value less than 0.05 was interpreted as significant.

\section{Results}

\section{Demographic and clinical data}

One hundred and ten (110) participants were recruited to the study - consisting of 66 subjects with diabetes (cases) and 44 subjects without diabetes (controls). For both cases and controls, over $80 \%$ were made up of females. The mean ages for cases and controls were $55.85 \pm 8.82$ years and $50.32 \pm 12.44$ years respectively (Table 1). Fasting plasma glucose concentration was higher among the cases compared with the controls $(p=0.000)$. In this study, cases were mostly on stable doses of Glibenclamide, Metformin and Insulin in various combinations. Among patients with diabetes, $24.2 \%(n=16)$ were on a combination of Glibenclamide and Metformin while $22.7 \%(\mathrm{n}=15)$ used both metformin and Insulin. About $40 \%(n=26)$ of cases were on metformin alone for the management of diabetes. Overall, 9 diabetic patients $(\mathrm{n}=$ 13.6) used other diabetic drugs aside metformin.

\section{Oxidative stress}

Table 2 shows the SOD activity values analyzed on blood samples obtained from the participants. Superoxide dismutase activity among case patients $(285.44 \pm 114.36 \mathrm{U} / \mathrm{ml})$ did not significantly differ ( $p$-value 0.144 ) from those observed within the control group $(319.79 \pm 126.12 \mathrm{U} / \mathrm{ml})$. When SOD activity was compared within case patients, we observed homogeneity in SOD activity values between patients using metformin only, those on diabetic medications aside metformin, and those taking metformin in combinations with other drugs (degree of freedom, 2; F-value, $0.261 ; p$-value, 0.771$)$.

\section{Haematological parameters}

Table 3 compares various haematological indices between diabetic patients and the control cohort. There was a significantly higher $(\mathrm{p}=0.023)$ total white blood cell count in the diabetes cohort compared to control subjects. There did not appear to be differences in lymphocyte count, neutrophil count, red cell count, platelet count, haemoglobin and haematocrit levels between diabetic cases and the control group.

\section{Correlation between SOD and haematological parameters}

In Table 4, association between SOD activity and haematological changes are compared. Higher neutrophil activity seemed to weakly correlate with increased SOD activity $\left(R=0.249 ; R^{2}=6.2 \% ; p<0.049\right)$. None of the other measured haematological indices correlated with SOD activity values. Similarly, there did not appear to be correlation between FBG and SOD activity.

Table 1 Summary of clinical parameters in diabetic subjects in comparison to controls

\begin{tabular}{lll}
\hline Parameters & Number of cases & Number of controls \\
\hline Number & 66 & 44 \\
Female/male & $55 / 11$ & $38 / 6$ \\
Mean Age (years) \pm SD & $55.85 \pm 8.82$ & $50.32 \pm 12.44$ \\
FBG (mmol/L) \pm SD & $8.34 \pm 3.47$ & $4.75 \pm 0.55$ \\
Duration of Diabetes (years) \pm SD & $10.67 \pm 7.53$ & - \\
Anti-hypertensive drugs & $39(59.0 \%)$ & $8(18.1 \%)$ \\
Patients on Metformin only & $26(39.4 \%)$ & - \\
Patients on Metformin plus other drugs & $57(83.6 \%)$ & - \\
Patients Metformin plus Glibenclamide & $16(24.2 \%)$ & - \\
Patients Metformin plus Insulin & $15(22.7 \%)$ & - \\
Patients on other drugs aside metformin & $9(13.6)$ & -
\end{tabular}


Table 2 Superoxide dismutase activity in diabetic subjects in comparison to controls

\begin{tabular}{|c|c|c|c|}
\hline Parameters & Cases \pm SD & Controls \pm SD & $p$ value \\
\hline Total SOD Activity (U/ml) & $285.44 \pm 114.36$ & $319.79 \pm 126.12$ & 0.144 \\
\hline SOD Activity $(\mathrm{U} / \mathrm{ml})$ of cases on Metformin only ${ }^{a}$ & $274.81 \pm 122.73$ & - & $a^{*} b^{*} c=0.771$ \\
\hline SOD Activity $(\mathrm{U} / \mathrm{ml})$ of cases on Metformin and other drugs ${ }^{b}$ & $281.75 \pm 111.91$ & - & Post hoc: \\
\hline \multirow[t]{3}{*}{ SOD Activity $(\mathrm{U} / \mathrm{ml})$ of cases on other drugs aside Metformin ${ }^{c}$} & $307.98 \pm 133.40$ & & $a * b=0.824$; \\
\hline & & & $a^{*} c=0.499$ \\
\hline & & & $b^{*} c=0.5265$ \\
\hline
\end{tabular}

FBG- Fasting blood glucose; SOD-Superoxide dismutase; SD, standard deviation

\section{Discussion}

Oxidative stress is increasingly being reported as a widely accepted participant in the progression of diabetes and its complications. In this study, the mean fasting plasma glucose level of patients with diabetes was $8.34 \mathrm{mmol} / \mathrm{l}$ (Table 2) - significantly higher than the recommended target FPG indicative of good glycaemic control (3.9 - $7.2 \mathrm{mmol} / \mathrm{l})$ - and confirming that the cases were more likely to be exposed to persistent hyperglycaemia [10, 11]. Persistent hyperglycaemia in diabetes is often accompanied by increased production of free radicals or impaired antioxidant defenses [7, $12-14]$. In the present study, the SOD activity tended to be lower in the diabetes group ( $p>0.05$ however) compared to controls but the trend did not achieve statistical significance. Majority of the diabetic cases were on metformin (either as monotherapy or in combination with other medications such as insulin) and most likely may have benefited from their antioxidant properties. Metformin, a drug taken to control diabetes has been reported to possess antioxidant properties by inhibiting generation of reactive oxidative species in diabetes and thereby reducing oxidative stress [15]. A reduction in ROS levels in human leukocytes due to metformin administration by direct scavenging of the free radicals has been reported by Bonnefont-Rousselot et al. [16]. Other hypoglycaemic agents such as insulin have also been shown to have similar impact on oxidative stress [17].

Table 3 Full blood count of study participants

\begin{tabular}{llll}
\hline Parameters & Cases \pm SD & Controls \pm SD & $p$ value \\
\hline WBC $\left(\times 10^{3} / \mu \mathrm{L}\right)$ & $5.87 \pm 1.76$ & $5.25 \pm 1.05$ & $0.023^{*}$ \\
RBC $\left(\times 10^{6} / \mu \mathrm{L}\right)$ & $4.48 \pm 0.60$ & $4.57 \pm 0.54$ & 0.426 \\
HGB $(\mathrm{g} / \mathrm{dL})$ & $12.29 \pm 1.50$ & $12.60 \pm 1.30$ & 0.273 \\
HCT $(\%)$ & $38.77 \pm 5.01$ & $39.32 \pm 3.29$ & 0.485 \\
Platelets $\left(\times 10^{3} / \mu \mathrm{L}\right)$ & $233.89 \pm 62.81$ & $221.05 \pm 59.62$ & 0.286 \\
NEU $(\%)$ & $46.73 \pm 14.44$ & $47.89 \pm 10.64$ & 0.649 \\
LYM $(\%)$ & $44.33 \pm 14.43$ & $43.80 \pm 9.50$ & 0.816 \\
\hline
\end{tabular}

WBC-White blood cells; RBC-Red blood cells; HGB-Haemoglobin; HCT-Haematocrit; NEU-Neutrophils; LYM-Lymphocytes (* $p$-value $<0.05$ is considered significant); $\mathrm{SD}$, standard deviation
Changes in haematological indices, except for the higher WBC trend in the diabetes group, were similar in both study cohorts. It is noteworthy that the high white cell count in the diabetes group is in keeping with the increased oxidative stress triggered by the high levels of hyperglycaemia observed in this cohort. Polymorphonuclear and mononuclear leukocytes can be activated by advanced glycation end-products and cytokines in a state of hyperglycaemia [18].

Our study reports of SOD activity that significantly albeit weakly correlated with neutrophil count. The observation agree well with findings by Kotani and Sakane [19] who worked on diacron reactive oxygen metabolites (d-ROMs) as clinical markers for evaluation of total oxidative burden. Their work showed positive correlation between neutrophil counts and d-ROMs levels in asymptomatic female subjects. Neutrophils secrete a variety of inflammatory cytokines such as interleukin- 6 which trigger an increase in oxidative stress [20,21]. Oxidative stress also activates the inflammatory pathway with leukotriene B4 which attract neutrophils to inflammatory sites further generating oxidative stress $[19,22]$.

Table 4 Correlation between SOD Activity Levels and haematological indices

\begin{tabular}{|c|c|c|}
\hline Pearson's Correlations & $\mathrm{R}$ & $p$ value \\
\hline FBG and SOD Levels & -0.044 & 0.727 \\
\hline WBC and SOD Levels & 0.047 & 0.714 \\
\hline RBC and SOD Levels & 0.113 & 0.375 \\
\hline HGB and SOD Levels & 0.028 & 0.824 \\
\hline HCT and SOD Levels & 0.002 & 0.99 \\
\hline MCV and SOD Levels & -0.092 & 0.469 \\
\hline MCH and SOD Levels & -0.078 & 0.542 \\
\hline MCHC and SOD Levels & 0.004 & 0.975 \\
\hline PLT and SOD Levels & 0.235 & 0.061 \\
\hline NEU(\%) and SOD Levels & $0.249^{*}$ & $0.049^{*}$ \\
\hline LYM(\%) and SOD Levels & -0.208 & 0.099 \\
\hline \multicolumn{3}{|c|}{$\begin{array}{l}\text { *Correlation is significant at } p \text { value }<0.05 \text { (2-tailed) } \\
\text { WBC-White blood cells; RBC-Red blood cells; HGB-Haemoglobin; HCT-Haematocrit; } \\
\text { NEU-Neutrophils; LYM-Lymphocytes; FBG- Fasting blood glucose; SOD- Superoxide } \\
\text { dismutase; PLT-platelet count; NEU-neutrophil count; LYM- lymphocyte count; } \\
\text { MCV- Mean corpuscular volume; MCH- Mean corpuscular haemoglobin; MCHC- } \\
\text { Mean corpuscular haemoglobin concentration }\end{array}$} \\
\hline
\end{tabular}


There are some potential limitations of this study that should be discussed briefly. First, the limited sample size. This could partly explain our findings of similar SOD activity values in the diabetic and control cohorts. A more large-scale survey is likely to be more accurate with little bias for unequal drug preferences. Another noteworthy limitation to our study is its cross-sectional design involving single measurement of haematological indices which may not demonstrate a temporal relationship with oxidative stress. Additionally intercurrent infections which were not actively sought for by our study cannot be completely excluded and might have potentially exerted confounding effects on the haematological indices.

\section{Conclusion}

In conclusion, we report similar SOD activity and for that matter similar oxidative stress levels in diabetic and non-diabetic adults. Oxidative stress as measured by SOD activity did not appear to correlate with FBG and several other haematological parameters. Further study would be required to investigate the relationship between these haematological indices and diabetic micro and macrovascular complications in our population.

\begin{abstract}
Abbreviations
DM: Diabetes Mellitus; ROS: reactive oxygen species; NDMRC: National Diabetes Management and Research Centre; SOD: Superoxide dismutase GPX: glutathione peroxidase; CAT: catalase; GSH: reduced gluthathione; DNA: Deoxyribonucleic acid; OGTT: Oral-Glucose-Tolerance-Test; HBA1C: glycated haemoglobin A1C; FPG: fasting plasma glucose; FBC: full blood count; Cu/ZnSOD: Copper/Zinc Superoxide dismutase; SPSS: Statistical Package for Social Sciences; O2--: superoxide; O2: oxygen; RBC: Red blood cells; d-ROMs: diacron reactive oxygen metabolites.
\end{abstract}

\section{Competing interests}

The author(s) declare that they have no competing interests.

\section{Authors' contributions}

ARH and APF conceived the study; participated in its design, coordination, and collation of laboratory data; and helped to draft the manuscript. $Y G, A H$, AS, ATN, BCA, AG and DB participated in the study design, coordination and collation of laboratory data; performed the statistical analysis; and helped to draft the manuscript. ADN participated in the study design and coordination and helped to draft the manuscript. AJ participated in the study design and coordination and helped to draft the manuscript. All authors read and approved the final manuscript.

\section{Acknowledgements}

We are most grateful to Noah Obeng-Nkrumah (successfulnoahforchrist@ yahoo.com) for the splendid review and editing. We thank the staff of National Diabetes Management and Research Centre (NDMRC) in Korle-Bu Teaching Hospital, Accra Ghana.

\section{Author details}

${ }^{1}$ Department of Medical Laboratory Sciences, School of Biomedical and Allied Health Sciences, University of Ghana, Korle-bu, Accra, Ghana. ${ }^{2}$ Department of Chemical Pathology, School of Biomedical and Allied Health Sciences, University of Ghana, Korle-bu, Accra, Ghana. ${ }^{3}$ Department of Medicine, School of Medicine and Dentistry, Korle-bu, Accra, Ghana. ${ }^{4}$ Department of Medical Biochemistry, School of Biomedical and Allied Health Sciences, University of Ghana, Korle-bu, Accra, Ghana.

Received: 11 March 2015 Accepted: 26 June 2015

Published online: 20 August 2015

\section{References}

1. International Diabetes Federation: IDF Diabetes Atlas. (2014) http:// www.diabetesatlas.org. Accessed on 16 December 2014

2. Ishikawa K, Takenaga K, Akimoto M. ROS-generating mitochondrial DNA mutations can regulate tumor cell metastasis. Science. 2008:320:661-4.

3. Halliwell B. Free radicals and antioxidants - quo vadis? Trends Pharmacol Sci. 2011;32(3):125-30

4. Malstrom B, Andreason L, Reinhammer B. XIIB. In: Boyer P, editor. The Enzymes. New York: Academic; 1975. p. 533-42.

5. Bertini I, Magnani S, Viezzoli MS. Structure and properties of copper-zinc superoxide dismutases. In: SAG, editor. Advances in Inorganic Chemistry. New York: Academic; 2008. p. 127-250.

6. Erejuwa OO. Oxidative Stress in Diabetes Mellitus: Is There a Role for Hypoglycemic Drugs and/or Antioxidants? In: Lushchak V, editor. Oxidative Stress and Diseases. London: Academic; 1990. p. 217-46.

7. Comazzi S, Spagnolo V, Bonfanti U. Erythrocyte changes in canine diabetes mellitus: in vitro effects of hyperglycaemia and ketoacidosis. J Comp Clin Path. 2004;12:199-205.

8. Olson H, Betton G, Robinson D, Thomas K, Monro A, Kolaja G, et al. Concordance of thetoxicity of pharmaceuticals in humans and in animals. Toxicolo Pharmacol. 2000;32:56-67.

9. American Diabetes Association Position Statement. Standards of Medical Care in Diabetes. Diabetes Care. 2014;37(1):S15-23.

10. Marshall WJ, Bangert SK. Disorders of carbohydrate metabolism. In: Marshall WJ, Bangert SK, editors. Clinical Chemistry. China: Mosby/Elsevier Limited; 2004. p. 197-8.

11. Hoffbrand AV, Moss PAH, Pettit JE. Essential haematology: erythropoiesis and general aspects of anaemia. 5th ed. London: Blackwell Publishing; 2006

12. Rice-Evans C, Omorphos SC, Baysal E. Sickle cell membranes and oxidative damage. Biochem J. 1986;237:265-9.

13. Halliwell B, Gutteridge JM. Role of free radicals and catalytic metal ions in human disease: An overview. Meth Enzymol. 1990;186:81-5.

14. Ceriello A. "Postprandial hyperglycemia and diabetes complications: Is it time to treat?". Diabetes. 2005;54:1-7.

15. Rosen P, Wiernsperger NF. Metformin delays the manifestation of diabetes and vascular dysfunction in Goto-Kakizaki rats by reduction of mitochondrial oxidative stress. Diabetes/Metabolism Research Reviews. 2006:22:323-30.

16. Bonnefont-Rousselot D, Raji B, Walrand S. An intracellular modulation of free radical production could contribute to the beneficial effects of metformin towards oxidative stress. Metab Clin Exp. 2003;52:586-9.

17. Tuzcu H, Aslan I, Aslan M. The Effect of High-Dose Insulin Analog Initiation Therapy on Lipid Peroxidation Products and Oxidative Stress Markers in Type 2 Diabetic Patients. Oxidative Med Cell Longev. 2013;2013:1-9.

18. Chung FM, Shin SJ, Tsai JC, Lee YJ, Chang DM. Peripheral total and differential leucocyte count in diabetic nephropathy. Diabetes Care. 2005:28(7):1710-7.

19. Kotani K, Sakane N. White Blood Cells, Neutrophils and Reactive Oxygen Metabolites among Asymptomic Subjects. Int J Prev Med. 2012;2(3):428-31.

20. Kasama T, Miwa Y, Isozaki T, Odai T, Adachi M, Kunkel SL. Neutophil-derived cytokines: Potential therapeutic targets of inflammation. Curr Drug Targets Inflamm Allergy. 2005:4:273-9.

21. Saliu JA, Elekofehinti OO, Komolafe K, Oboh G. Effects of some green leafy vegetables on the haematological parameters of diabetic rats. J Nat Prod Plant Resour. 2012;2(4):482-5

22. Thannickal VJ, Fanburg BL. Reactive oxygen species in cell signalling. Am J Physiol Lung Cell Mol Physiol. 2000;279:L1005-28.

\section{Submit your next manuscript to BioMed Central and take full advantage of:}

- Convenient online submission

- Thorough peer review

- No space constraints or color figure charges

- Immediate publication on acceptance

- Inclusion in PubMed, CAS, Scopus and Google Scholar

- Research which is freely available for redistribution 